\title{
Ultra biomicroscopy findings in goniotomy-assisted transluminal trabeculotomy - a case report
}

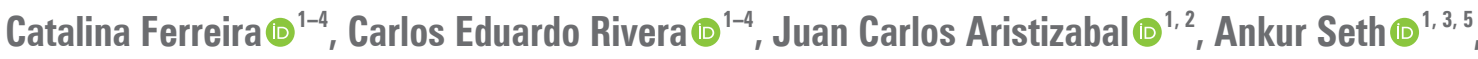 \\ Edgar Muñoz (1) \\ ${ }^{1}$ Collective Innovations Colombia, Cali, Colombia \\ ${ }^{2}$ Pontificia Universidad Javeriana, Cali, Colombia \\ ${ }^{3}$ GSR Medical Center, Cali, Colombia \\ ${ }^{4}$ GSR International, United States \\ ${ }^{5}$ University of Tennessee Health Science Center, United States
}

\begin{abstract}
BACKGROUND: Goniotomy-assisted transluminal trabeculotomy (GATT) is a minimally invasive technique that avoids conjunctival incision and is guided by light through the Schlemm's canal using a microcatheter with an illuminated tip. This technique decreases intraocular pressure (IOP) by improving flow through the Schlemm's canal. We present two cases of glaucoma patients who underwent GATT surgery for IOP control.

CASES PRESENTATION: The first case is a 19-year patient with juvenile glaucoma that underwent GATT because of uncontrolled IOP with a successful outcome. The second case is a 64-year female patient with primary open-angle glaucoma who underwent GATT surgery because of uncontrolled IOP who presented a cyclodialysis secondary to the procedure, with an adequate IOP after surgery. Ultra biomicroscopy (UBM) was used to assess the anatomical changes associated with surgery.

CONCLUSIONS: Goniotomy-assisted transluminal trabeculotomy is a safe technique but not free of risks and potential complications. Ultra biomicroscopy is a diagnostic aid that allows us to provide valuable information to evaluate the pre-surgical, post-surgical anatomy and possible complications to follow-up and guide the management in required cases.
\end{abstract}

KEY WORDS: glaucoma; intraocular pressure; goniotomy-assisted transluminal trabeculotomy

Ophthalmol J 2021; Vol. 6, 178-183

\section{INTRODUCTION}

Glaucoma is the leading cause of irreversible blindness worldwide. It is estimated that $3.54 \%$ of the population in the world has this disease [1]. Although effective in many cases, medical treatment represents a challenge, mainly due to poor adher- ence, drug toxicity, and, in developing countries, the costs and access to medications are common barriers.

An alternative to conventional trabeculectomy or drainage devices for glaucoma that represents serious risks and complications for patients is minimally invasive glaucoma surgery. It is a safer option,

CORRESPONDING AUTHOR:

Carlos Rivera, Holguines Trade Center, Cra 10 11-60, office 236, tel: (2) 3165225961, (572) 5546567; e-mail: carlosriverahoyos@gmail.com, crivera@grupogsr.co

This article is available in open access under Creative Common Attribution-Non-Commercial-No Derivatives 4.0 International (CC BY-NC-ND 4.0) license, allowing to download articles and share them with others as long as they credit the authors and the publisher, but without permission to change them in any way or use them commercially 
which helps decrease intraocular pressure (IOP) and reduces dependence on medications [1].

Goniotomy-assisted transluminal trabeculotomy (GATT), described by Grover et al. in 2014 [2], reduces IOP, increasing the flow of aqueous humor through the Schlemm's canal and collector channels. It is carried out with an illuminated microcatheter or a prolene 5-0 that helps identify and cannulate the Schlemm's canal in 360 degrees. Once the catheter is in position, it is pulled, causing a rupture of the trabecular meshwork. This technique uses an ab interno approach, is sutureless, and utilizes a conjunctival sparing technique for possible future interventions. It can be done alone or in combination with cataract surgery.

Although the treatment is 360 degrees of the angle, it is not possible in some cases. It can be limited between 180 to 300 degrees, with successful results leading to reduced IOP at 12 months follow-up [3].

Initially, GATT was indicated for primary congenital glaucoma, but recently, it has been used successfully for mild to moderate glaucoma in adults with open-angle glaucoma and juvenile glaucoma $[2,4,5]$.

The most frequent complication during the first week is hyphema that resolves spontaneously [4]. Other potential complications are flattening of the anterior chamber, choroidal folds due to hypotonia, choroidal effusion, and vitreous hemorrhage $[4,6]$.

In the postoperative period, after resorption of the hyphema, a posterior leaflet of the trabeculae appears, called the trabecular shelf, which sometimes adheres to the peripheral iris and is seen protruding on it. This is indicative of a good surgical result $[2,4]$.

GATT surgery in developing countries may be limited due to high associated costs. Long-term follow-up is necessary to verify if there is a closure of drainage because of scarring of the Schlemm's canal.

In this study, we present outcomes in two postoperative clinical cases of GATT with ultra-biomicroscopic findings.

\section{CASE 1}

A 19-years-old man presented to the clinic with a 3-year diagnosis of juvenile glaucoma. The patient had been treated with brimonidine, dorzolamide, and timolol. The visual acuity (VA) of the right eye (RE) was 20/25 and in the left eye (LE) was hand movement. In the RE, he had an IOP of $13 \mathrm{~mm}$
$\mathrm{Hg}$, open angles, and a cup/disc ratio of 0.95 excavation with superior and inferior rim thinning.

In the LE, he had an afferent pupillary defect, IOP of $34 \mathrm{~mm} \mathrm{Hg}$, open angles, and a cup/disc ratio of $100 \%$.

It was decided to perform a GATT surgery in the LE in order to control the IOP.

In the postoperative period (POP), he presented with a hyphema that was reabsorbed in less than a week, with no sequelae. Intraocular pressure has stabilized at $12 \mathrm{~mm} \mathrm{Hg}$ without medication after a year of follow-up.

In Figure 1, the ultrasound biomicroscopy shows the trabecular shelf after GATT surgery.

Patient data is not identifiable.

\section{CASE 2}

A 64-year-old female presented to the clinic with a diagnosis of primary open-angle glaucoma. The patient had received maximum medical treatment with brimonidine, dorzolamide, timolol, and latanoprost.

The corrected VA in the RE was finger counting and in the LE was 20/25.

In the RE, she had a dense cataract, the IOP was $26 \mathrm{~mm} \mathrm{Hg}$, open angle, and cup/disc ratio of 0.8 with a double notch.

In the LE, the IOP was $14 \mathrm{~mm} \mathrm{Hg}$ and a cup/disc ratio of 0.6 with a slight inferior rim thinning.

Because she presented a cataract with a poorly controlled IOP despite a maximum medical treatment, it was decided to perform a combined (GATT) surgery and cataract extraction by phacoemulsification.

During the catheter insertion in the trabecular meshwork, an obstruction was felt after it passed the first quadrant and the illumination tip was not visible, so it was decided to stop the procedure and remove the catheter.

On the 4th day after surgery, she presented an IOP of $5 \mathrm{~mm} \mathrm{Hg}$ and a $100 \%$ hyphema that prevented properly assessing the anterior and posterior segment.

A RE ultrasound was performed (Fig. 2-5): a hyphema was occupying almost all of the anterior chamber, the intraocular lens in the capsular bag, a plane ciliochoroidal detachment, a nasal cyclodialysis, and a cystic space in the temporal ciliary body, which was associated with a false route of the microcatheter. 


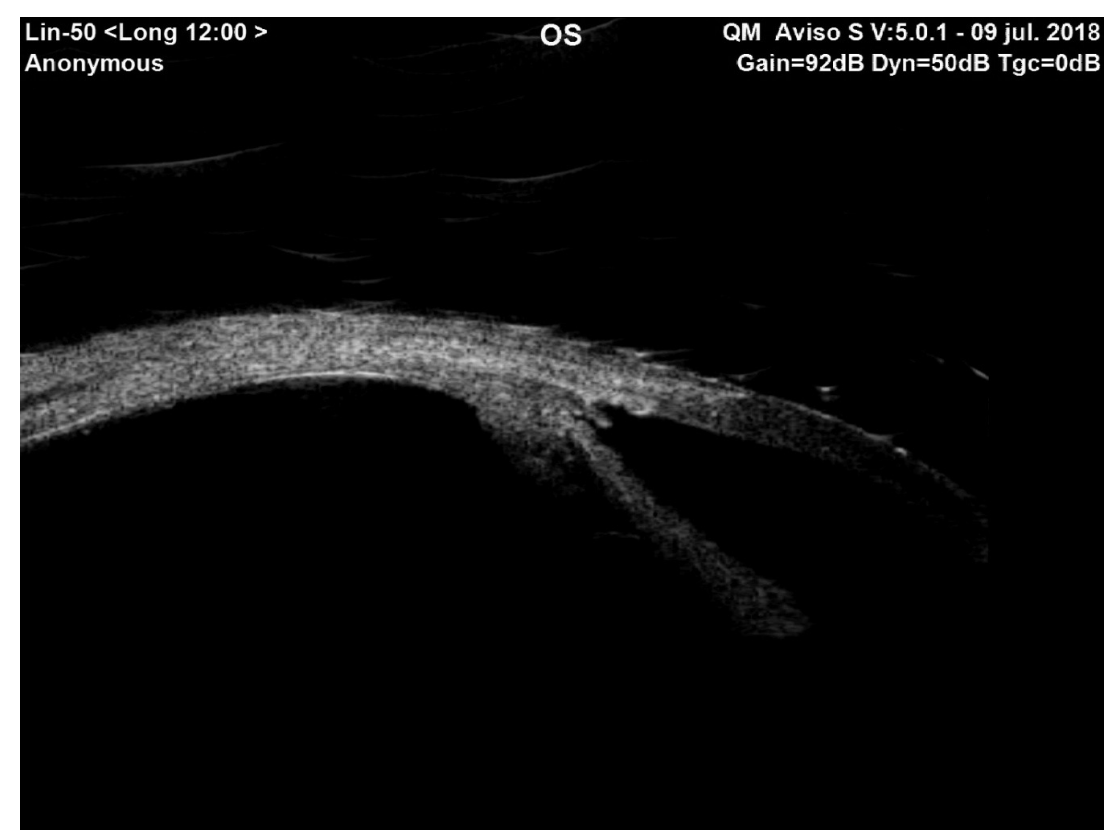

FIGURE 1. Ultra biomicroscopy (UBM): Iongitudinal section of the anterior chamber angle in a patient after a goniotomy-assisted transluminal trabeculotomy (GATT). There is an open Schlemm's canal and trabecular shelf

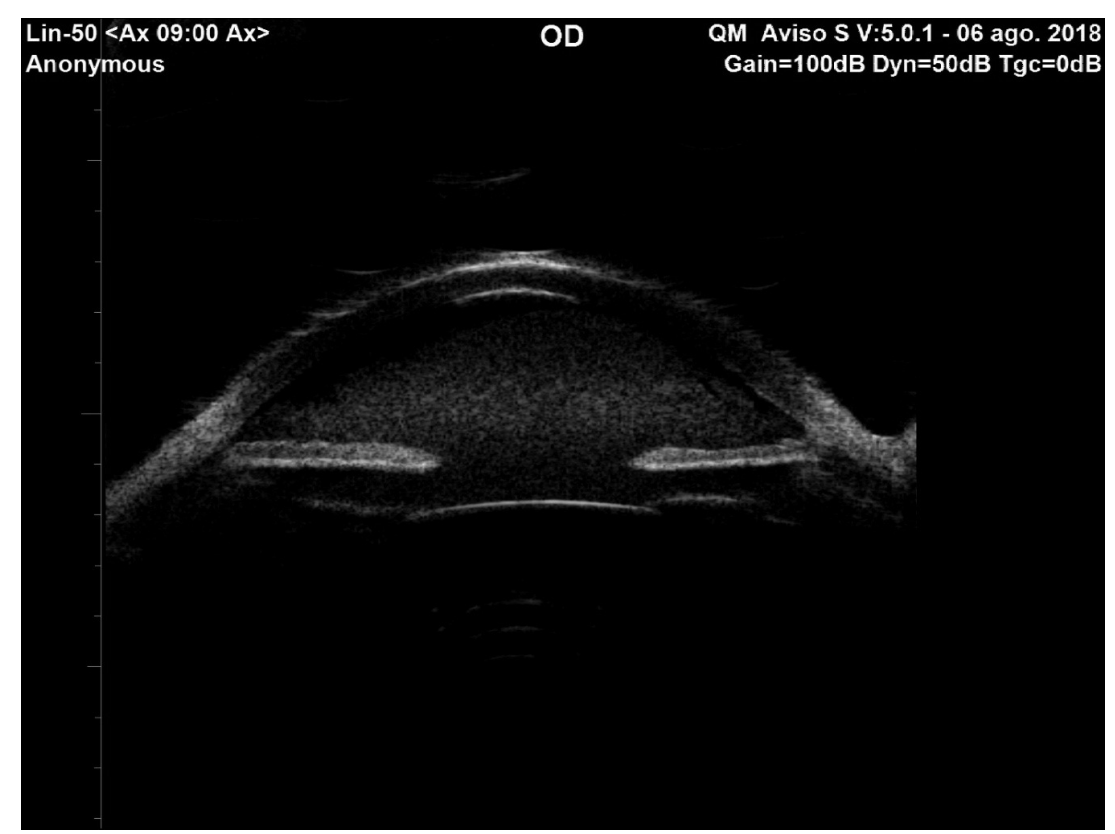

FIGURE 2. Ultra biomicroscopy (UBM): axial section. Intraocular lens in adequate position and a hyphema that occupies a large part of the anterior chamber

Management was started with cyclopentolate $1 \%$, three times a day, and at day 14 th POP, total reabsorption of the hyphema was found, with an IOP in $10 \mathrm{~mm} \mathrm{Hg}$ without medication. In the LE, the IOP was $19 \mathrm{~mm} \mathrm{Hg}$ with maximum medical treatment.
The corrected VA in the RE was 20/30 and in the LE was 20/25. The patient had a follow-up of 2 years where the IOP remains at $10 \mathrm{~mm} \mathrm{Hg}$. Apparently, the limited cyclodialysis helped control the IOP.

Patients' data are not identifiable. 


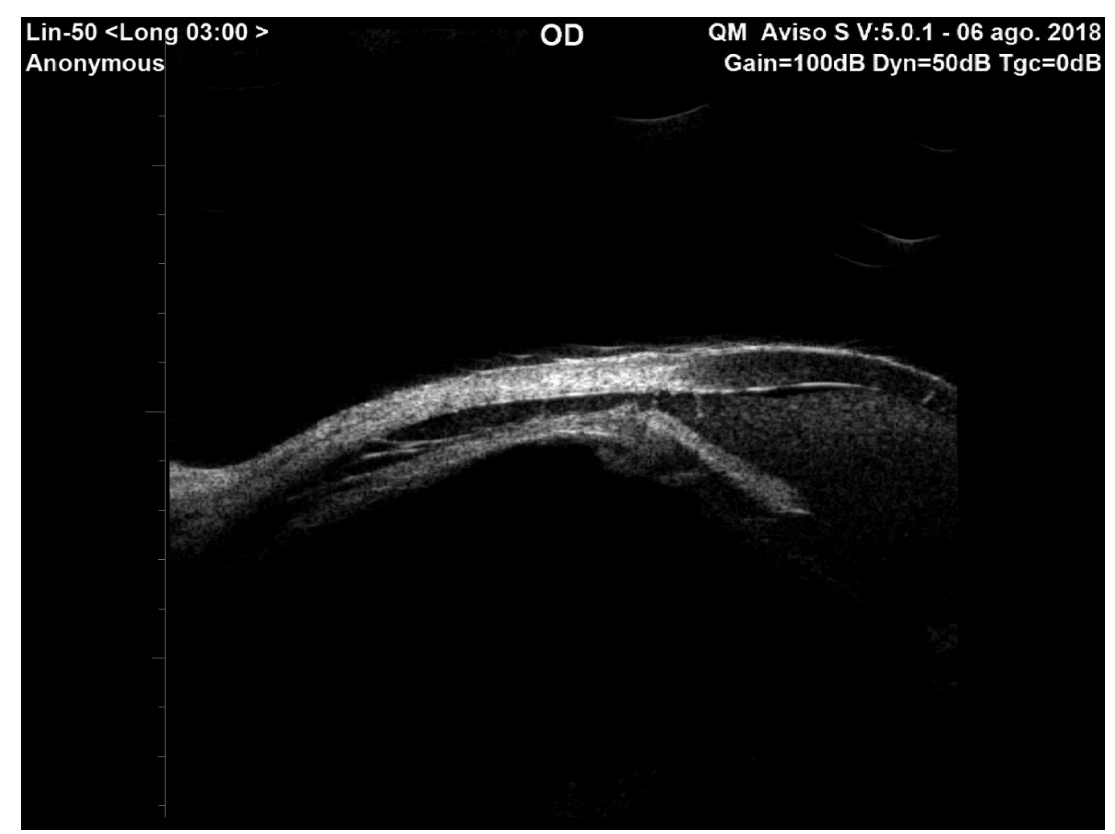

FIGURE 3. Ultra biomicroscopy (UBM): Iongitudinal section showing an iridodialysis, a ciliary body detachment, and a supraciliary effusion

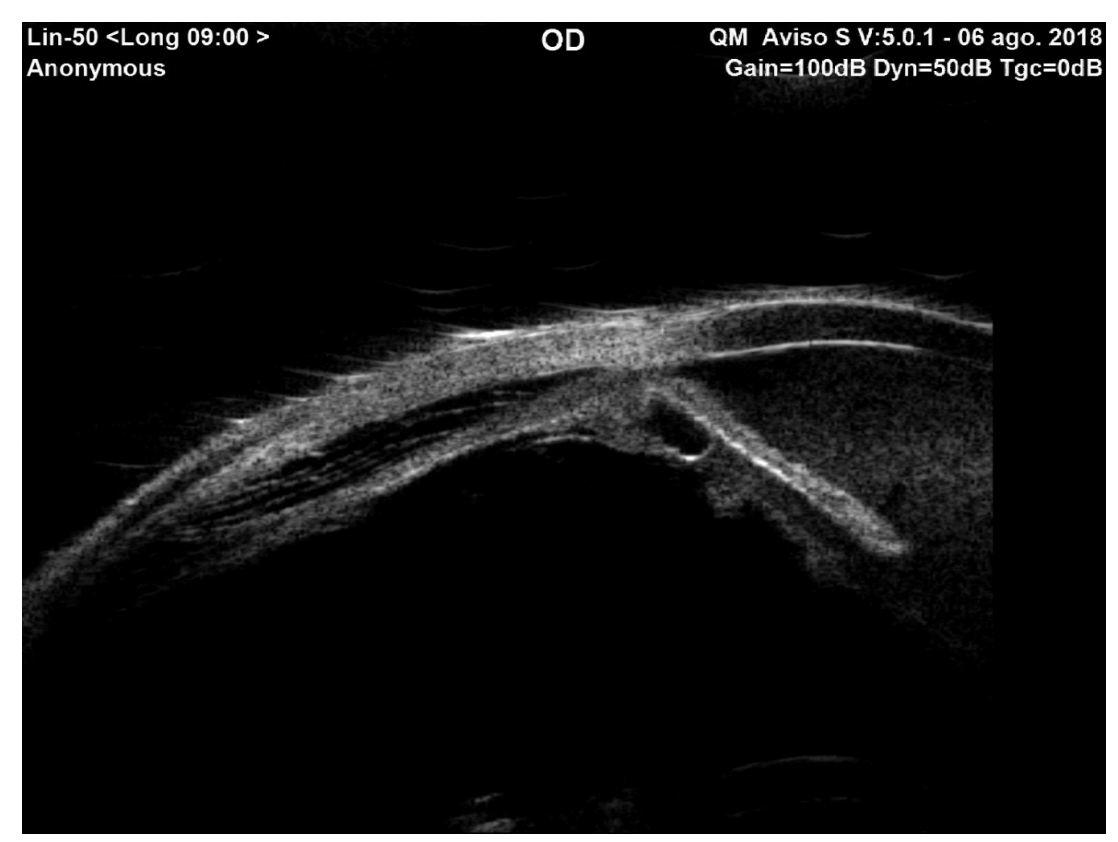

FIGURE 4. Ultra biomicroscopy (UBM): Iongitudinal section. Hyphema and a cystic space in the ciliary body associated with a cyclodialysis and a uveal effusion. It is likely that a misdirected catheter produces this cyst

\section{DISCUSSION}

Goniotomy-assisted transluminal trabeculotomy is a minimally invasive glaucoma surgery with an internal approach that improves the aqueous outflow through the Schlemm's canal and is based on the fact that the juxtacanalicular system is the point of the greatest resistance to flow in most cases of open-angle glaucoma [1]. It does not require a conjunctival or scleral incision and is safe and effective. Goniotomy-assisted transluminal trabeculotomy has a success rate between $68 \%$ and $90 \%$ in cases of open-angle glaucoma in children and adults [4].

Despite the advantage represented by the illumination of the catheter and that the success 


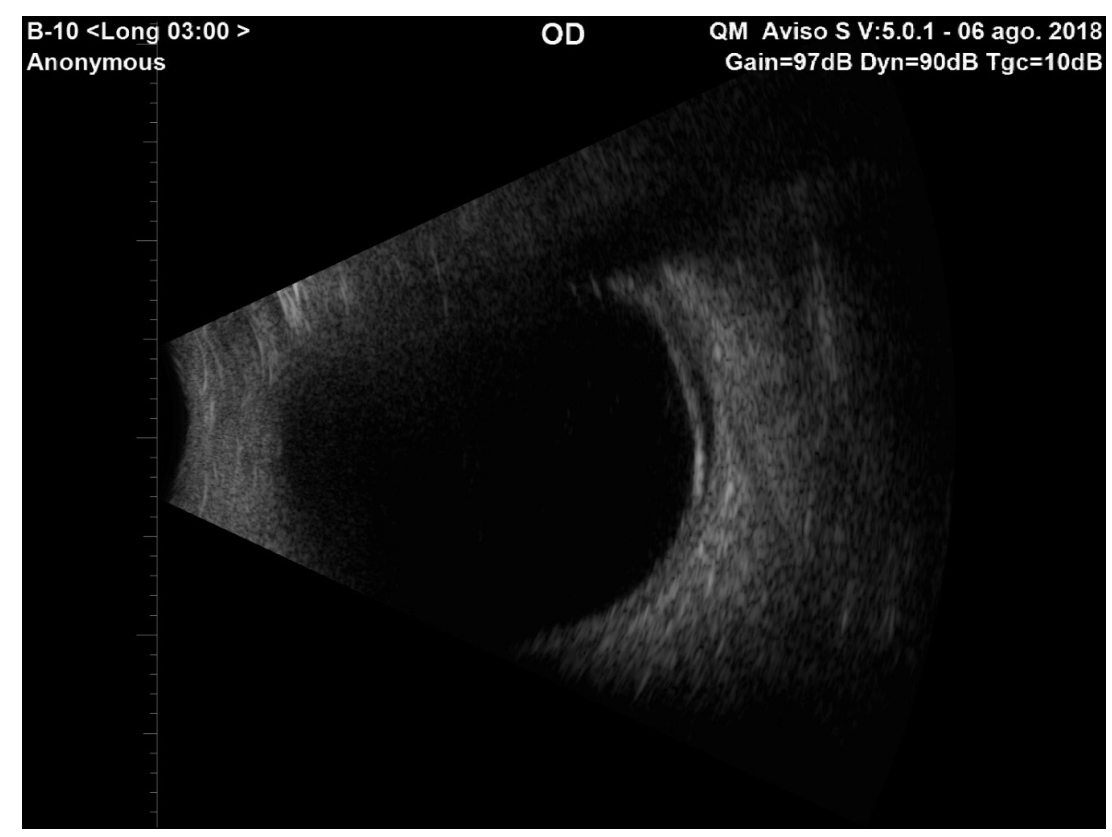

FIGURE 5. B-mode ultrasound with a $10 \mathrm{MHz}$ probe in which a choroidal flat detachment is shown. Space in the ciliary body is associated with a cyclodialysis and a uveal effusion. A misdirected catheter likely produces this cyst

rate is high, the possibility of complications, such as misdirection of the catheter, can occur and have serious consequences depending on the direction taken [2].

Fortunately, thanks to the illuminated tip, it is noteworthy that it is easy to notice misdirection, in which case it can be retracted and repositioned. A posterior direction of the light should alert to a possible unnoticed passage to the sub choroid or subretinal space $[7,8]$.

Ultra biomicroscopy (UBM) can be used as a diagnostic tool to evaluate the pre- and post-operative anatomy. It allows visualizing complications with refractive media opacity to search for causes and provide adequate management and follow-up to patients.

UBM should be used as an elective technique in patients with suspected post-surgical or post-traumatic cyclodialysis or iridodialysis. It allows evaluation of the lesions, delimits lateral and anteroposterior extensions describing detailed choroidal compromise. The morphology of the angle and ciliary body is clearly observed, ruling out associated pathology [9].

Cyclo- and iridodyalisis can be underdiagnosed conditions or go unnoticed, being treated empirically or having spontaneous regression, mainly in cases of opaque media [9].

\section{CONCLUSION}

Goniotomy-assisted transluminal trabeculotomy surgery has a relatively long learning curve, which requires at least 5 to 10 procedures and is not free of risks and complications. The surgeon who wants to learn should handle the anatomy of the anterior chamber angle, angle of surgery and be sufficiently trained to operate with gonioprism [4]. Absolute contraindications for the procedure must be taken into account, which are the use of anticoagulants that cannot be suspended, unstable IOP, angular closure, severe endothelial compromise, or poor visualization of the trabecular meshwork [4]. Ultra biomicroscopy is a diagnostic aid that allows us to provide valuable information to evaluate the pre-surgical, post-surgical anatomy and possible complications to follow up and give guidance on the management in required cases.

\section{REFERENCES}

1. Richter GM, Coleman AL. Minimally invasive glaucoma surgery: current status and future prospects. Clin Ophthalmol. 2016; 10: 189-206, doi: 10.2147/0PTH.S80490, indexed in Pubmed: 26869753.

2. Grover D, Godfrey D, Smith O, et al. Gonioscopy-Assisted Transluminal Trabeculotomy, Ab Interno Trabeculotomy. Ophthalmology. 2014; 121(4): 855-861, doi: 10.1016/j.ophtha.2013.11.001, indexed in Pubmed: 24412282.

3. Shi Y, Wang H, Yin J, et al. Microcatheter-assisted trabeculotomy versus rigid probe trabeculotomy in childhood glaucoma. Br J Ophthalmol. 2016; 100(9): 1257-1262, doi: 10.1136/bjophthalmol-2015-307880, indexed in Pubmed: 26674778. 
4. Grover DS, Smith O, Fellman RL, et al. Gonioscopy assisted transluminal trabeculotomy: an ab interno circumferential trabeculotomy for the treatment of primary congenital glaucoma and juvenile open angle glaucoma. Br J Ophthalmol. 2015; 99(8): 1092-1096, doi: 10.1136/ bjophthalmol-2014-306269, indexed in Pubmed: 25677669.

5. Lim ME, Dao JB, Freedman SF. 360-Degree Trabeculotomy for Medically Refractory Glaucoma Following Cataract Surgery and Juvenile Open-Angle Glaucoma. Am J Ophthalmol. 2017; 175: 1-7, doi: 10.1016/j.ajo.2016.11.011, indexed in Pubmed: 27916715.

6. Shakrawal J, Bali S, Sidhu T, et al. Randomized Trial on IlluminatedMicrocatheter Circumferential Trabeculotomy Versus Conventional Trabeculotomy in Congenital Glaucoma. Am J Ophthalmol.
2017; 180: 158-164, doi: 10.1016/j.ajo.2017.06.004, indexed in Pubmed: 28624326.

7. Sarkisian SR. An illuminated microcatheter for 360-degree trabeculotomy [corrected] in congenital glaucoma: a retrospective case series. J AAPOS. 2010; 14(5): 412-416, doi: 10.1016/j.jaapos.2010.07.010, indexed in Pubmed: 21035067.

8. Verner-Cole EA, Ortiz S, Bell NP, et al. Subretinal suture misdirection during 360 degrees suture trabeculotomy. Am J Ophthalmol. 2006; 141(2): 391-392, doi: 10.1016/j.ajo.2005.08.038, indexed in Pubmed: 16458706.

9. Koga-Nakamura, W. Ultrabiomicroscopía y ciclodiálisis. Rev Mex Oftalmol. 2007; 81(6): 326-333. 